\title{
Metagenomic Analysis of the Airborne Environment in Urban Spaces
}

\author{
Nicholas A. Be • James B. Thissen • Viacheslav Y. Fofanov • \\ Jonathan E. Allen • Mark Rojas • George Golovko • Yuriy Fofanov • \\ Heather Koshinsky • Crystal J. Jaing
}

Received: 8 May 2014 / Accepted: 9 October 2014 / Published online: 29 October 2014

(C) The Author(s) 2014. This article is published with open access at Springerlink.com

\begin{abstract}
The organisms in aerosol microenvironments, especially densely populated urban areas, are relevant to maintenance of public health and detection of potential epidemic or biothreat agents. To examine aerosolized microorganisms in this environment, we performed sequencing on the material from an urban aerosol surveillance program. Whole metagenome sequencing was applied to DNA extracted from air filters obtained during periods from each of the four seasons. The composition of bacteria, plants, fungi, invertebrates, and viruses demonstrated distinct temporal shifts. Bacillus thuringiensis serovar kurstaki was detected in samples known to be exposed to aerosolized spores, illustrating the potential utility of this approach for identification of intentionally introduced microbial agents. Together, these data demonstrate the temporally dependent metagenomic complexity of urban aerosols and the potential of genomic analytical techniques for biosurveillance and monitoring of threats to public health.
\end{abstract}

Electronic supplementary material The online version of this article (doi:10.1007/s00248-014-0517-z) contains supplementary material, which is available to authorized users.

N. A. Be $\cdot$ J. B. Thissen $\cdot$ C. J. Jaing $(\bowtie)$

Physical and Life Sciences Directorate, Lawrence Livermore

National Laboratory, Livermore, CA 94551, USA

e-mail: jaing2@llnl.gov

V. Y. Fofanov $\cdot$ H. Koshinsky

Eureka Genomics, Hercules, CA, USA

J. E. Allen

Computation/Global Security Directorates, Lawrence Livermore

National Laboratory, Livermore, CA, USA

M. Rojas $\cdot$ G. Golovko $\cdot$ Y. Fofanov

Sealy Center for Structural Biology and Molecular Biophysics,

University of Texas Medical Branch, Galveston, TX, USA
Keywords Aerosol microbiology · Urban air · Airborne bacteria $\cdot$ Metagenomics $\cdot$ Microbiome

\section{Introduction}

Airborne microbial communities have not been as well characterized as organisms present in soil or aquatic environments [1-4], in part due to the difficulty associated with obtaining sufficient material for study. Previous studies have performed the culture-based examination of airborne microbial populations relevant to agricultural environments $[5,6]$, city settings $[7,8]$, and meteorological conditions $[9,10]$. Many aerosolized organisms may, however, be difficult to culture, and there has been an increasing interest and an expanding technical capacity for metagenomic surveys of aerosol environments. A number of such studies have been applied to aerosol microbes with potential human contact, such as those in indoor spaces [11-13], suburban and city regions [14, 15], and industrial food and waste facilities [16-18]. These studies have revealed that microbial composition varies depending on many factors, including time, location, and human and animal behavior.

Aerosolized microorganisms are also capable of transcontinental spread $[19,20]$. While not all transported organisms may affect human health, recent studies suggest the possibility that transoceanic aerosols could bear etiologic agents of human disease [21]. Aerosolized microorganisms would be difficult or impossible to control, particularly in densely populated urban areas; thus, analytical protocols capable of surveying these populations are of interest for public health and national defense.

Biosurveillance technologies are an important part of the national strategy to prevent the dissemination of a pathogenic biological agent. Toward this end, the U.S. Department of Homeland Security has been operating the BioWatch program, a network of systems for environmental monitoring 
[22]. BioWatch collects samples from ambient air and is designed to function as an early warning apparatus for local officials and public health authorities. We sought to leverage this collection framework to perform a seasonal survey of the microbial aerosol communities associated with the national capital area. An additional interest of this study was to determine the efficacy of next-generation sequence analysis for identifying a particular agent within an aerosol background. We therefore examined collection filters known to be exposed to Bacillus thuringiensis serovar kurstaki spores, which are commonly applied as an insecticide against gypsy moths [23], to determine whether $B$. thuringiensis could be detected during dispersal periods.

We used whole metagenome next-generation sequencing to evaluate the sequence content captured by aerosol collectors as comprehensively as possible. While the majority of previous environmental studies have applied $16 \mathrm{~S}$ ribosomal DNA-based sequencing approaches, we used whole metagenome sequencing to increase the degree of taxonomic resolution and facilitate the identification of nonchromosomal sequences, which could improve species-level characterization and identify viruses, fungi, and plants.

\section{Methods}

\section{Extraction and Purification from BioWatch Filters}

Archived portable sampling unit (PSU) filters were obtained from the Washington D.C. National Capital Region. Filters were collected every day during a 1-week period from each season: winter (January 22-28, 2009), spring (April 20-26, 2009), summer (July 19-25, 2009), and fall (October 25-31, 2009). Filters were also obtained on a day during which B. thuringiensis serovar kurstaki spores were actively dispersed as a pesticide (May 3, 2007). Eleven filters (corresponding to 11 sampling unit locations) were extracted from every day of the 1-week sampling period, with the exception of the winter, during which only seven of the above sampling unit locations were available for this study. Filters from all locations throughout the 1-week period were combined for extraction. The same locations were surveyed throughout the study period. Filters for some additional locations beyond the 11 noted above were available; however, filters obtained from these locations were obscured by soot, likely due to proximity of the sampling unit to particulate-generating activities. In these cases, the filters could not be used due to the inhibitory effects of particulate matter on DNA amplification. The relative quantity of such soiled filters did not vary according to season. Up to 24 filters were combined per $50-\mathrm{mL}$ conical tube. Thirty milliliters of $100 \mathrm{mM}$ phosphate buffer ( $\mathrm{pH} 7.4$ ) with $0.05 \%$ Tween 80 was added to each tube. Samples were vortexed for $30 \mathrm{~s}$ and placed on a rocking shaker for $15 \mathrm{~min}$.
The vortexing and rocking process was repeated, in the same buffer, for three additional times. Filters were removed, and the washing buffer was centrifuged to collect the filter material.

DNA purification was performed on the collected pellet using the UltraClean Soil DNA Isolation Kit (MoBio) with some modifications. The pellet extracted from combined filters was resuspended in $100 \mu \mathrm{L}$ TE buffer, $350 \mu \mathrm{L}$ MoBio Bead Solution, $60 \mu \mathrm{L}$ MoBio Solution S1, and $200 \mu \mathrm{L}$ MoBio Inhibitor Removal Solution. The resuspended pellet was bead beat for $2 \mathrm{~min}$ with $0.5-\mathrm{mm}$ zirconia/silica beads and then centrifuged. To the removed supernatant, $250 \mu \mathrm{L}$ of MoBio Solution S2 was added, incubated at $4{ }^{\circ} \mathrm{C}$ for $5 \mathrm{~min}$, and centrifuged at $10,000 \times \mathrm{g}$ for $1 \mathrm{~min}$. Two volumes of MoBio Solution S3 were added to the supernatant. The solution was added to a MoBio spin filter and centrifuged for $1 \mathrm{~min}$ at $10,000 \times g$, followed by three washes with $300 \mu \mathrm{L}$ MoBio Solution S4. DNA samples were eluted with $50 \mu \mathrm{L}$ MoBio Solution S5.

\section{DNA Amplification}

DNA extracted from the samples was amplified using the REPLI-g Midi Kit (Qiagen). This kit performs whole genome amplification using multiple displacement amplification [24, 25]. Samples were amplified for $16 \mathrm{~h}$ at $30{ }^{\circ} \mathrm{C}$. Amplified samples were purified using QIAquick PCR Purification columns (Qiagen).

\section{Sequencing and Quality Control}

Two independent sets of the amplified seasonal samples (four samples in each set) were prepared into libraries and sequenced. Each amplified DNA sample in the first set was sequenced on the Illumina Genome Analyzer IIx (GAIIx), using $1 \mu \mathrm{g}$ DNA for preparation of standard paired-end libraries. Briefly, DNA was fragmented, end repaired, $\mathrm{A}^{\prime}$ tagged, ligated to adaptors, size selected, and enriched with 18 PCR cycles (the spring sample was enriched with 13 cycles of PCR). Each amplified DNA sample in the second set was sequenced on the Illumina HiSeq 2000, using 300 ng DNA for preparation of paired-end, indexed libraries, as described above.

The spring sample from the first set of the seasonal samples was sequenced on the GAIIx using 51-bp paired-end reads, while the summer, fall, and winter samples from the first set were sequenced as 112-bp paired-end reads on the GAIIx (one sample per lane). All the seasonal samples from the second set were sequenced using 50-bp single-end reads on the HiSeq 2000. Equivalent amounts of the four libraries were multiplexed in one flow cell lane.

Samples exposed to $B$. thuringiensis serovar kurstaki were sequenced using 51-bp paired-end reads on the GAIIx. The 
resultant sequence reads were processed using the default parameters of the Illumina CASAVA pipeline and checked for quality issues. All reads were determined to be of sufficient quality to proceed with subsequent analysis.

\section{Sequence Read Analysis}

Genomic composition of each sample was determined by mapping its sequence reads against viral, bacterial, and eukaryotic (excluding human) sequences (NCBI GenBank database as of March 1, 2013) using Bowtie (version 0.12.7) with up to three mismatches. Because a top-hit-only approach carries a risk of producing false-positive hits (read mapping to a relative of the organism present in the sample, rather than the actual organism), all hits (up to three mismatches) produced by the alignment program were kept and analyzed.

The resulting output from each Bowtie run was parsed to obtain the taxonomy IDs and the names of the organisms that were matched by each read. All possible hits for each read were recorded and classified on the basis of their taxonomic classification using the NCBI taxonomic ID. To avoid bias resulting from overrepresentation of certain species within GenBank and bias associated with reads present in multiple copies within a genome (e.g., rDNA reads), a read was counted as matching to a given taxonomic ID only once, preventing the artificial inflation of number of mapped reads from species with multiple available sub-strain genomes.

To improve specificity, an additional analysis was performed to identify reads whose alignments were unique to a single species - referred to as "informative reads." This was particularly helpful in distinguishing between reads mapping to closely related species sharing significant sequence similarity. Additionally, when multiple sub-strains of a given bacterial species were present, the bacterial or viral sub-strains were collapsed, such that reads mapping to sub-strains were instead counted as mapping to parent species. This prevented the erroneous dilution of unique reads for species with many sub-strains.

\section{Quantitative PCR for B. thuringiensis Serovar kurstaki}

The genome copy number for B. thuringiensis serovar kurstaki present on aerosol filters was quantified using Taqman (Life Technologies) quantitative PCR (F: AGCGTA TGCTCGTCTCAAGTAAAA, R: CCTGCCTTGTGGATCT CTAGC, probe: TGCATCGAACTCAATAAAATATTTGT TTTGGAGGG). A standard curve was constructed ranging from 1 to 100,000 genome copies using B. thuringiensis serovar kurstaki HD1 genomic DNA and was applied for absolute quantification of the experimental samples. All assays were performed in triplicate.
Statistical Analysis

The Phyloseq (version 1.6.0) package in R (version 3.0.2) was used for data manipulation and construction of graphics [26]. Principal coordinate analysis-based ordering was used to construct heat maps and ordination plots with the Phyloseq implementation of NeatMap [27]. For each ordination plot, percentage variation explained by each component is given along the axes.

\section{Results}

Alignment of Urban Airborne Sequence Data

Sequence data obtained from the extracted, amplified aerosol samples, including total reads, unique reads, and informative mapped reads, are given for each seasonal sample in Table 1. Informative reads were defined as unique reads mapping to only one unique taxonomic ID. Informative reads were applied for rank-ordering taxonomic IDs. When assigning relative abundance values to an organism in subsequent analyses, total reads mapped to that taxonomic ID were employed in order to capture the total sequence data derived from that species.

Informative read values were comparable between the winter, summer, and fall samples but were observed to be much higher in the samples collected during the spring. This observation was primarily due to elevated levels of plant/fungal sequence material and the larger genome sizes associated with these organisms. Specifically, the increased quantity of informative reads was almost entirely due to mapping of sequence data to Betula nana (dwarf birch shrub), to which more than ten million informative reads were assigned in each spring sample.

Seasonal Distribution of Urban Airborne Sequence Data

The distribution of total sequence data (total mapped reads) was examined according to the category of origin (Fig. 1). As was expected, bacterial sequences were featured prominently in these data and observed at the highest levels during the summer, followed by the winter. Sequences associated with plant/fungal material were also frequently observed, peaking during the spring. Sequence data mapping to invertebrate genomes were highest during the summer, remaining relatively consistent in the remainder of the year. Reads were also mapped to phage, sequences annotated as synthetic constructs, vertebrates, and other viruses; however, the sequence content and the number of mapped informative reads were minimal for each of these categories throughout all seasons. 
Table 1 The number of total and unique reads sequenced and the number of informative reads aligned to taxonomic IDs. Informative reads represent unique reads mapping to only one taxonomic ID

\begin{tabular}{llllr}
\hline Sample & $\begin{array}{l}\text { Total reads } \\
\text { sequenced }\end{array}$ & $\begin{array}{l}\text { Unique reads } \\
\text { sequenced }\end{array}$ & $\%$ unique & $\begin{array}{l}\text { Informative } \\
\text { reads mapped }\end{array}$ \\
\hline Winter 1 & $90,454,366$ & $21,722,517$ & 24.01 & $3,727,579$ \\
Winter 2 & $64,234,662$ & $18,759,050$ & 29.2 & $2,123,026$ \\
Spring 1 & $85,253,374$ & $66,947,821$ & 78.53 & $19,124,779$ \\
Spring 2 & $68,588,917$ & $57,647,167$ & 84.05 & $15,703,546$ \\
Summer 1 & $97,944,883$ & $31,861,677$ & 32.53 & $4,096,208$ \\
Summer 2 & $51,686,698$ & $23,524,936$ & 45.51 & $2,657,978$ \\
Fall 1 & $97,854,175$ & $55,640,354$ & 56.86 & $4,053,402$ \\
Fall 2 & $68,228,814$ & $45,999,051$ & 67.42 & $2,246,574$ \\
\hline
\end{tabular}

A union set of the top 15 detected genomes, within each season, was compiled and rank ordered based on the number of mapped informative reads (Table 2). This smaller union set was selected to more closely examine the highest abundant organisms present throughout the course of the year. The majority of the top-ranked genomes corresponded to bacteria, largely attributable to soil microbiota. Species commonly associated with skin microflora, including Staphylococcus and Klebsiella, were also prominently observed. B. nana was a dominant contributor to the observed plant sequence data, though this was primarily observed during the spring and fall. The peak in sequence data mapping to invertebrates during the summer was attributed largely to Aedes aegypti. Due to the whole metagenome approach, a unique feature of this study was the ability to survey viral content collected by aerosol filters. Although Pseudomonas phage was the only virus identified in the top-ranked organisms, a total of 60 viruses were assigned sequence data in this study (Electronic Supplementary Material (ESM) Fig. S1). It is important to note, however, that very few informative reads were assigned to these taxa, indicating that a portion of these alignments may be attributable to other organisms.

\section{Species-Based Clustering of Seasonal Samples}

Samples from each season were grouped according to the relative abundance of their respective associated species, as measured using the relative number of reads mapped to each taxonomic ID (read counts were normalized to total sequence data for each sample). Clustering was performed using
Fig. 1 Total sequence data obtained from aerosol material from the four seasons. Material from urban aerosol collection sites was collected, and nucleic acid was extracted and amplified. Whole metagenome sequencing was performed in duplicate, and reads were mapped to bacterial, viral, plant, fungal, and eukaryotic (excluding human) reference genomes. Total reads mapped across both replicates are shown combined in the plot, which is segmented according to organism category. Quantity of sequence data (absolute abundance) is shown for each individual season within each category

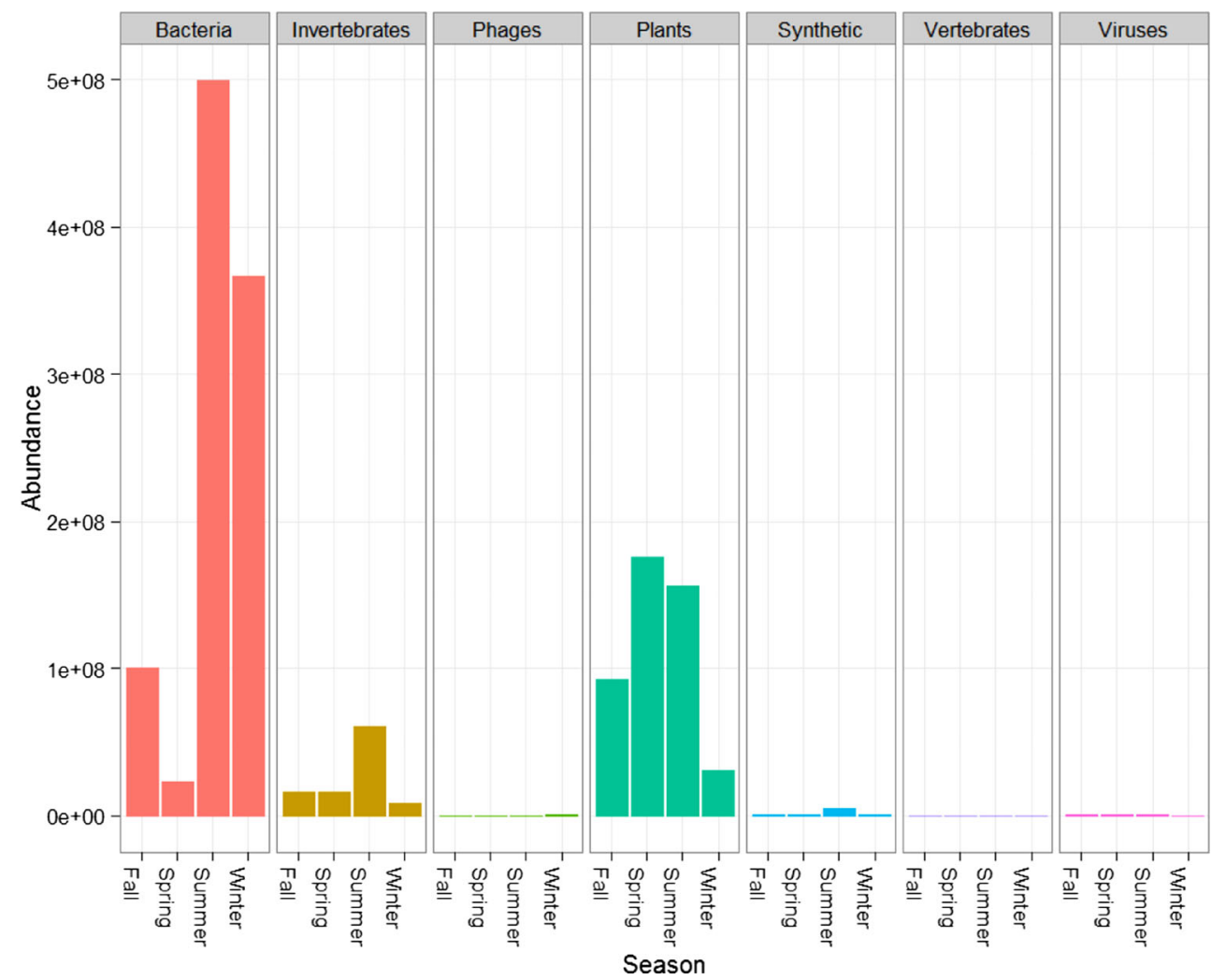


Table 2 A union set of the top 15 genomes to which sequence reads were aligned. Overall ranking of species is shown according to the number of informative reads mapped. Informative reads are defined as unique reads mapping to only one taxonomic ID

\begin{tabular}{|c|c|c|c|c|c|c|c|c|}
\hline \multirow[t]{3}{*}{ Species } & \multicolumn{8}{|c|}{ Ranking relative to all detected organisms (ranked by informative read count) } \\
\hline & \multicolumn{2}{|c|}{ Spring } & \multicolumn{2}{|l|}{ Fall } & \multicolumn{2}{|c|}{ Winter } & \multicolumn{2}{|c|}{ Summer } \\
\hline & 1 & 2 & 1 & 2 & 1 & 2 & 1 & 2 \\
\hline \multicolumn{9}{|l|}{ Bacteria } \\
\hline Bacillus cereus & 94 & 24 & 25 & 25 & 19 & 17 & 10 & 8 \\
\hline Bacillus clausii & 220 & 224 & 64 & 67 & 150 & 78 & 12 & 15 \\
\hline Bacillus coagulans & 115 & 109 & 53 & 51 & 55 & 51 & 4 & 3 \\
\hline Bacillus megaterium & 30 & 29 & 9 & 8 & 3 & 2 & 2 & 1 \\
\hline Bacillus psychrosaccharolyticus & 138 & 142 & 98 & 96 & 6 & 5 & 26 & 27 \\
\hline Bacillus pumilus & 165 & 157 & 28 & 29 & 40 & 36 & 9 & 11 \\
\hline Bacillus smithii & 143 & 149 & 30 & 32 & 20 & 18 & 5 & 5 \\
\hline Bacillus sp. 1NLA3E & 262 & 305 & 189 & 156 & 9 & 6 & 35 & 32 \\
\hline Bacillus subtilis & 353 & 365 & 142 & 115 & 238 & 204 & 15 & 16 \\
\hline Bacillus thuringiensis & 209 & 49 & 66 & 60 & 72 & 89 & 20 & 14 \\
\hline Beta proteobacterium FWI2 & 52 & 61 & 19 & 22 & 13 & 14 & 27 & 49 \\
\hline Bradyrhizobium elkanii & 50 & 78 & 68 & 110 & 10 & 15 & 104 & 275 \\
\hline Cupriavidus basilensis & 6 & 6 & 1 & 1 & 1 & 1 & 1 & 4 \\
\hline Cupriavidus necator & 40 & 59 & 17 & 24 & 11 & 11 & 22 & 40 \\
\hline Cupriavidus sp. HMR-1 & 12 & 14 & 7 & 7 & 2 & 3 & 6 & 10 \\
\hline Geobacillus caldoxylosilyticus & 68 & 68 & 40 & 39 & 15 & 16 & 3 & 2 \\
\hline Klebsiella pneumoniae & 38 & 45 & 15 & 20 & 8 & 8 & 13 & 20 \\
\hline Ralstonia pickettii & 8 & 11 & 6 & 6 & 4 & 4 & 7 & 13 \\
\hline Ralstonia solanacearum & 21 & 31 & 8 & 13 & 7 & 10 & 18 & 34 \\
\hline Staphylococcus epidermidis & 14 & 13 & 120 & 104 & 234 & 514 & 17 & 9 \\
\hline Staphylococcus hyicus & 88 & 85 & 407 & 2749 & 263 & 1799 & 21 & 12 \\
\hline Stenotrophomonas maltophilia & 197 & 260 & 34 & 37 & 14 & 12 & 28 & 26 \\
\hline \multicolumn{9}{|l|}{ Invertebrate } \\
\hline Aedes aegypti & 166 & 460 & 167 & 431 & 109 & 327 & 11 & 7 \\
\hline Hammondia hammondi & 29 & 44 & 41 & 55 & 5 & 7 & 63 & 103 \\
\hline Toxoplasma gondii & 220 & 301 & 127 & 114 & 98 & 82 & 14 & 17 \\
\hline \multicolumn{9}{|l|}{ Phage } \\
\hline Pseudomonas phage F10 & 727 & 2335 & 1572 & 2749 & 29 & 13 & 748 & 2666 \\
\hline \multicolumn{9}{|l|}{ Plant/fungal } \\
\hline Alternaria arborescens & 2 & 2 & 3 & 3 & 43 & 950 & 16 & 19 \\
\hline Aureobasidium pullulans & 3 & 3 & 22 & 23 & 230 & 866 & 110 & 166 \\
\hline Betula nana & 1 & 1 & 4 & 4 & 30 & 207 & 24 & 30 \\
\hline Botryotinia fuckeliana & 9 & 8 & 5 & 5 & 301 & 790 & 276 & 312 \\
\hline Castanea mollissima & 10 & 7 & 942 & 1173 & 940 & 1799 & 925 & 1015 \\
\hline Cedrus deodara & 582 & 639 & 10 & 9 & 734 & 1799 & 1189 & 2666 \\
\hline Chaetomium globosum & 26 & 30 & 14 & 16 & 12 & 9 & 57 & 78 \\
\hline Cladosporium sphaerospermum & 7 & 9 & 147 & 169 & 579 & 866 & 119 & 142 \\
\hline Cochliobolus sativus & 44 & 48 & 11 & 14 & 835 & 1799 & 32 & 38 \\
\hline Gibberella zeae & 11 & 10 & 162 & 135 & 763 & 1799 & 577 & 763 \\
\hline Ginkgo biloba & 23 & 15 & 248 & 194 & 1008 & 1799 & 1387 & 2666 \\
\hline Hordeum vulgare & 31 & 37 & 44 & 41 & 17 & 20 & 33 & 29 \\
\hline Oryza sativa & 15 & 17 & 86 & 91 & 73 & 81 & 186 & 180 \\
\hline Penicillium chrysogenum & 13 & 12 & 50 & 47 & 579 & 866 & 112 & 143 \\
\hline Pinus taeda & 4 & 4 & 16 & 12 & 445 & 1060 & 161 & 170 \\
\hline
\end{tabular}


Table 2 (continued)

\begin{tabular}{|c|c|c|c|c|c|c|c|c|}
\hline \multirow[t]{3}{*}{ Species } & \multicolumn{8}{|c|}{ Ranking relative to all detected organisms (ranked by informative read count) } \\
\hline & \multicolumn{2}{|c|}{ Spring } & \multicolumn{2}{|c|}{ Fall } & \multicolumn{2}{|c|}{ Winter } & \multicolumn{2}{|c|}{ Summer } \\
\hline & 1 & 2 & 1 & 2 & 1 & 2 & 1 & 2 \\
\hline Prunus persica & 17 & 16 & 13 & 10 & 802 & 790 & 532 & 433 \\
\hline Pyrenophora tritici-repentis & 42 & 46 & 12 & 11 & 608 & 1060 & 403 & 451 \\
\hline Quercus robur & 5 & 5 & 39 & 33 & 263 & 1799 & 258 & 530 \\
\hline Rhizopus microsporus & 436 & 489 & 21 & 15 & 1008 & 790 & 52 & 43 \\
\hline Sordaria macrospora & 22 & 22 & 2 & 2 & 128 & 128 & 19 & 18 \\
\hline
\end{tabular}

principal coordinate analysis-based ordering in Phyloseq. This grouping was applied both to the full dataset of all detected reference genomes (Fig. 2a) and to a union set of the top 15 taxonomic IDs detected across all seasons (Fig. 2b). Each member of this smaller union set is shown in Fig. 2c. In both cases, duplicates from each season cluster together. When only the top taxonomic IDs were considered, the observed dissimilarity between fall and winter samples was reduced, suggesting that high abundant organisms were more closely shared between these seasons.

Relative abundance within the individual bacterial, plant/ fungal, invertebrate, and viral categories (corresponding to $3496,2772,848$, and 60 detected taxa, respectively) was also examined, and sample ordination was performed separately for each category (Fig. 3). In each case, separation was less distinct than was the case when all taxa were examined simultaneously (Fig. 2), although bacterial data facilitated better overall resolution for each season. Specific species categories demonstrated different seasonal separations. For instance, abundance of viral taxa was highly distinct during the winter, while plant/fungal taxa abundance differed greatly during the spring and winter. Notably, the distance between seasons was greater than the distance between individual replicates in each case except virus/phage. These results illustrate that the degree of temporal resolution is dependent on the taxa studied and that combined examination of all available environmental sequence content facilitates higher resolution.

\section{Detection of B. thuringiensis Serovar kurstaki in Aerosol Filters}

Beyond performing a general survey of seasonal sequence content, an additional interest of this study was the capacity of whole metagenome sequence analysis for identification of a specific organism within an airborne environmental background. Material was obtained from aerosol filters known to be exposed to $B$. thuringiensis serovar kurstaki spores, a commonly employed insecticide, and sequencing was performed as previously. Counts for the top 15 detected taxonomic IDs observed in data from aerosol filters are given in ESM Fig. S2. Reads were assigned to multiple $B$. thuringiensis strains, including a large proportion mapping uniquely to B. thuringiensis serovar kurstaki. Overall, B. thuringiensis reads represented $21 \%$ of informative reads in aerosol filters. A large number of reads also mapped to Bacillus cereus, a close relative of $B$. thuringiensis. Quantitative PCR was performed to validate and quantify the presence of $B$. thuringiensis serovar kurstaki, yielding a $\mathrm{Ct}$ value of $22.6 \pm 0.1$. This value corresponded to a copy number of $6.01 \times 10^{4}$ genome copies per aerosol filter or $4.91 \times 10^{3}$ copies $/ \mathrm{cm}^{2}$. These data demonstrate the effective extraction and detection of $B$. thuringiensis serovar kurstaki spores.

\section{Discussion}

Airborne microbial communities bear relevance to both environmental and health safety concerns, particularly those populations associated with urban areas. The aerosol samples in this study allowed both for holistic examination of taxa and a case study for detection of a pathogen surrogate (B. thuringiensis serovar kurstaki) within an urban aerosol background. Current detection methods rely largely on multiplex PCR assay panels, which are designed to detect a specific subset of biological agents. This study demonstrates that analyses of shotgun metagenomic sequence data are capable of identifying specific pathogens of interest and could complement these existing technologies. In the case of a detection event, sequence data could provide supplemental information such as strain identity and antimicrobial resistance factors. Further, the unbiased whole metagenome approach allows for potential detection of unknown pathogens for which existing assays may not be designed.

To characterize the general airborne content across each of the four seasons, the material from aerosol filters collected from the National Capital Region during the winter, spring, 
Fig. 2 Grouping of airborne seasonal samples according to sequence content. Total sequence data obtained from each seasonal aerosol sample were normalized to obtain relative abundance values. Relative abundances were subjected to principal coordinate analysis, which was applied for grouping individual samples. a Ordination plot showing dissimilarity between all seasonal samples, as determined by relative abundance of all taxa. Percent variance is shown alongside each axis. $\mathbf{b}$ A union set composed of the top 15 identified taxa in each sample was constructed based on the number of mapped informative reads (unique read mapping to only one taxonomic ID). The ordination plot shows dissimilarity between seasonal samples, as determined using only the union set of high abundant taxa. c Heat map displaying only high abundant taxa. Samples are shown along the horizontal axis and taxa along the vertical axis
A

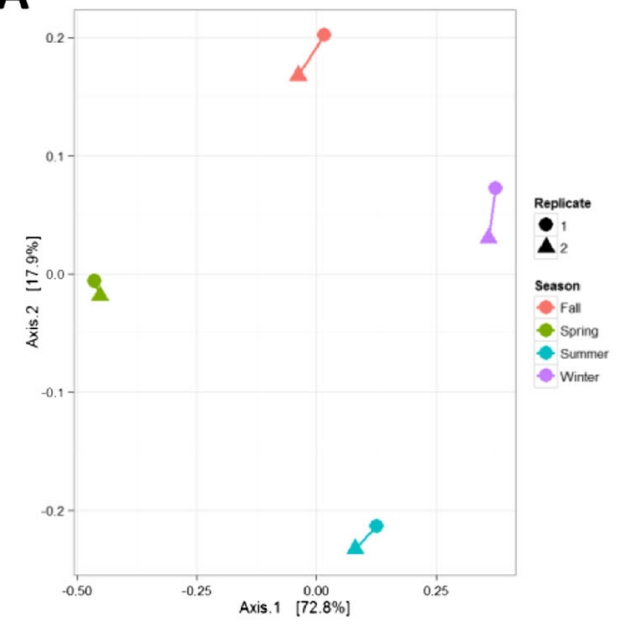

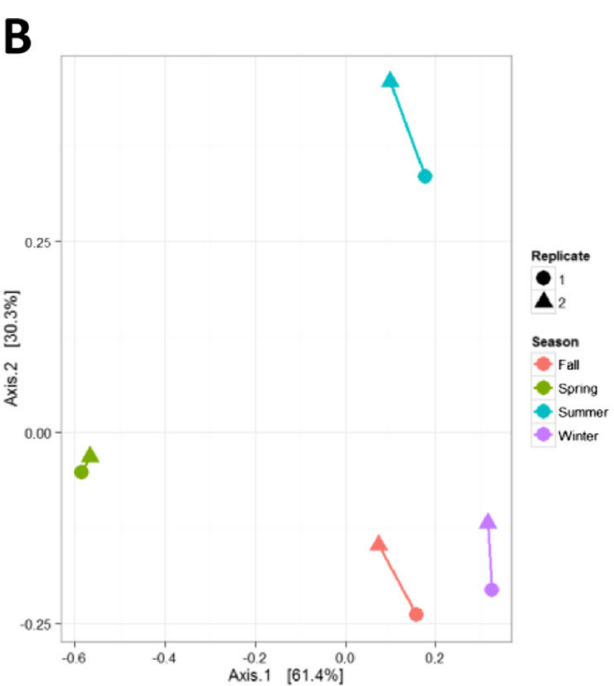

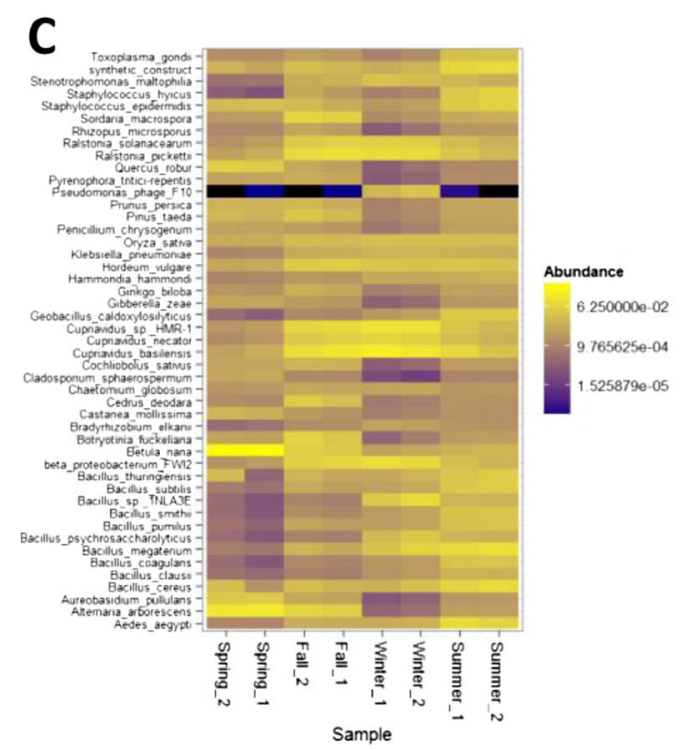

summer, and fall was extracted and sequenced. A whole metagenome approach was applied to capture all potential bacterial, viral, invertebrate, and plant sequences as well as to facilitate deeper taxonomic resolution.

Due to low available biomass, multiple displacement amplification (MDA) was performed prior to sequencing. Although whole genome amplification (WGA) does risk the introduction of bias in amplifying the microbial community, the MDA protocol used in this study has been demonstrated to be less biased in microarray-based microbial community analysis [24]. MDA-based protocols have been shown to result in less bias than other whole genome amplification methods, as determined by high-throughput sequencing of microbial genomes [28]. Commonly applied MDA kits have also been shown to result in uniform amplification through array-based analysis of DNA copy number variations [25]. It is, however, important to note that all amplification methods will impart some bias. Recent studies have demonstrated that WGA methods are influenced by DNA quality and size as well as GC content [29] and that observed microbial gene content may be biased by MDA [30]. While WGA, and specifically MDA, enables the unique examination of low biomass samples, experiments such as the studies cited above reinforce the importance of interpreting resultant data carefully, particularly when DNA integrity and GC content are expected to vary widely.

The ranking of bacterial taxa belonging to the genera Ralstonia, Cupriavidus, and Bacillus remained consistently high throughout the year, likely due to the fact that many of these organisms are ubiquitously found in soil. Among such species are Ralstonia pickettii, Ralstonia solanacearum, and Cupriavidus necator, which are not associated with human disease, although there have been reported cases of hospitalacquired $R$. pickettii infection [31]. Consistent observance of these organisms is likely due to continuous aerosolization from local soil reservoirs. 
Fig. 3 Similarity of airborne seasonal samples based on the sequence content from distinct taxonomic categories. For each seasonal sample, taxa and corresponding relative abundance values were segmented according to the following categories: bacteria, plant/fungal, invertebrate, and virus. Principal coordinate analysis was used to determine dissimilarity between samples, represented in ordination plots. Percent variance is given along each axis
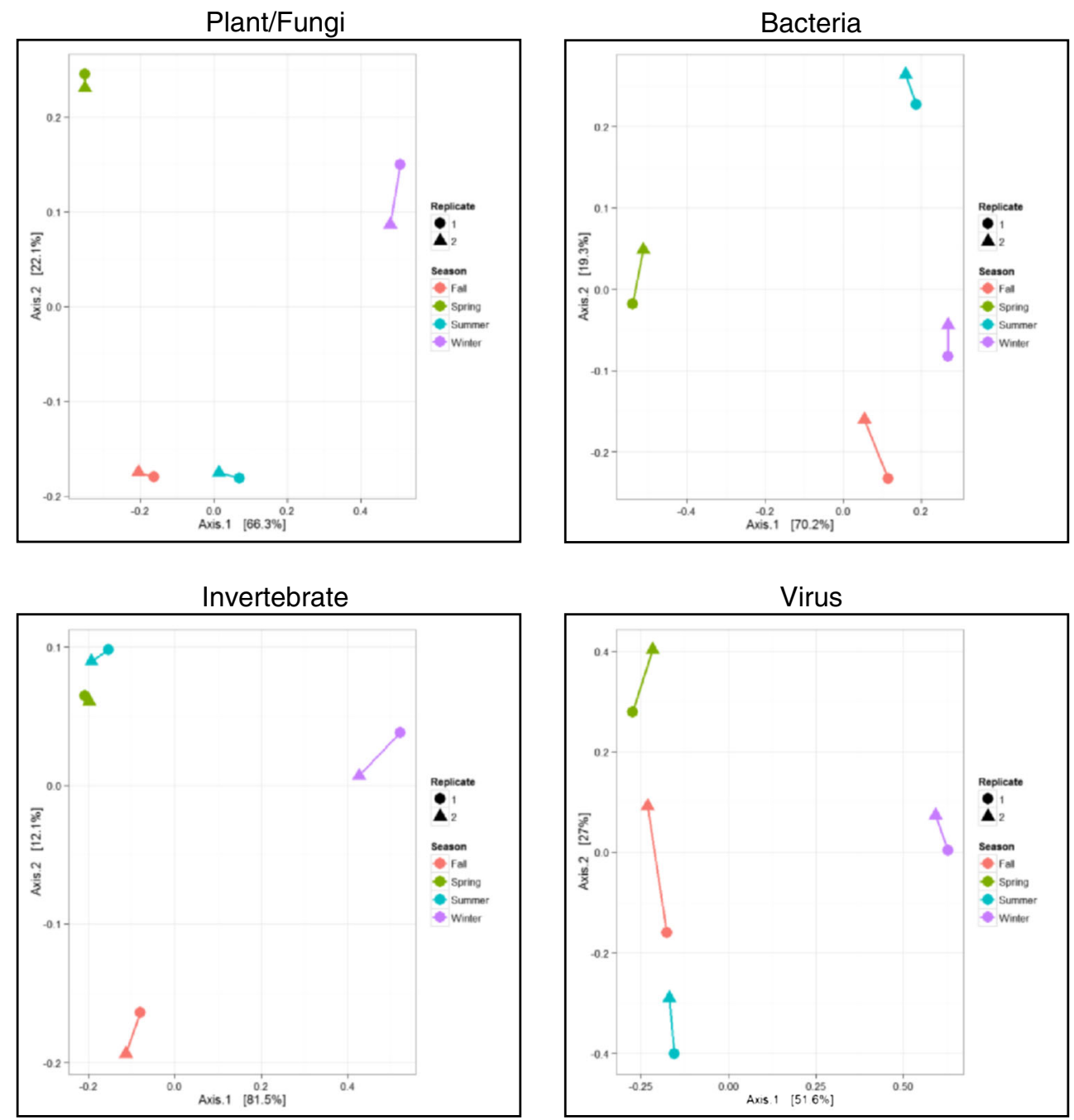

Members of the Bacillus genera, also commonly observed in soil, were similarly observed throughout the year. B. cereus and $B$. thuringiensis, both detected in this study, are closely related to Bacillus anthracis, the causative agent of anthrax [32]. Their presence could be highly relevant when attempting to identify $B$. anthracis specifically [33]. The increased frequency of $B$. thuringiensis during summer months is likely due to its noted insecticidal use. Many of the above noted bacteria maintained their presence during the winter months, despite the expected reduction in the aerosolization of microbe-laden terrestrial material. Previous studies have, however, observed elevated levels of certain bacterial groups during colder months, particularly those belonging to Bacteroides, Firmicutes, and Fusobacteria, which was attributed to aerosolized animal fecal material [15].

Staphylococcus ranking peaked during the summer and spring in our data, with comparatively low levels being observed during the winter and fall. Staphylococcus, in particular Staphylococcus epidermidis, are commonly found in the human skin microflora, with pathogenicity of staphylococcal infections varying widely dependent on strain, site of colonization, and host immune competence. Observation of Staphylococcus in urban aerosols could be due to aerosolization of human microflora. Reduced incidence during the winter and fall may be due to a limited epidermal exposure during colder months as well as a higher incidence of staphylococcal infections during the warm season [34].

Other primary contributors to sequence data obtained from urban aerosols included plants and fungi. This was particularly true during the spring, during which B. nana and Pinus taeda were the top-ranked plant taxa detected. These observations were expected, given increased levels of pollination during these time periods. Alternaria arborescens and Aureobasidium pullulans were the highest ranked fungal species detected and were likely collected simultaneously with associated plant material, as these organisms exist primarily as a plant pathogen and epiphyte, respectively.

The majority of observed viruses in this study were likely collected concurrently with specific bacterial or human content, such as bacteriophage and human-associated viral 
nucleic acid (herpesviruses, endogenous retroviruses). The seasonal patterns observed based on viral content may be reflective of these relationships. Given that our methodology was not expressly designed for viral particle collection, it is likely that a significant portion of airborne viruses were not isolated. A previous study specifically designed for collection of airborne viral content in the near-surface atmosphere did observe temporal patterns in viral composition, dependent on both temperature and humidity [35]. As expected, this previously published study yielded more extensive and diverse viral communities.

When taxonomic abundance data were employed for ordination of seasonal samples, distinct grouping of these samples was observed. It was also found that the use of a more taxonomically inclusive profile yielded improved seasonal resolution. Previous examination of the microbial composition in near-surface aerosols in the summer and winter similarly observed a correlation of samples by season [15] as well as according to land use characteristics at the collection location [14]. Further, recent work showed that the prevalence of rare, low-abundant taxa in a variety of environmental (including air) and clinical samples varied dramatically over time, disproportionately contributing to temporal changes in diversity [36]. Such seasonal correlation and annual recurrence of microbial communities have also been observed in remote, high altitude regions [37]. Spatial information was not available for the samples in this study; however, geographically dependent variations in the airborne microbial community structure have been previously observed [14, 38]. Observations from these studies, in combination with the temporal clustering observed in the current study, suggest the possibility that metagenomic profiles could potentially be useful for tracing samples with distinct origins.

In addition to assessing general aerosol content, a further aim of this study was to determine whether intentional aerosol release of an organism could be detected by metagenomic sequence analysis. Toward this end, the material known to be exposed to the aerosolized pesticide, B. thuringiensis serovar kurstaki, was subjected to sequencing. Sequence analysis did detect the presence of $B$. thuringiensis, confirming the capacity of our metagenomic sequence analysis approach for detecting a spore-borne aerosolized biological agent. In the actual event of detection of such an agent, further assays such as quantitative PCR could be performed for subsequent validation.

A sequencing-based approach may not be practical in all instances due to the extended time required for characterization. Further, although low biomass samples may be effectively examined via WGA with nanogram starting quantities of DNA, these techniques are costly to perform. It is, therefore, likely that sequencing would be applied as a complementary approach or if higher resolution analysis is required for detailed genomic inquiry. Sequencing throughput and costs are, however, scaling rapidly; thus, it is likely that the described protocols could approach standard practice with technical advancement and analytical automation for specific applications.

In summary, our data represent a broad seasonal survey of an urban aerosol bioburden, providing a temporal indication of the airborne metagenomic complexity in this space. Such data could be valuable in discerning whether a detected organism or profile is distinct from the microbial background. The ability to survey the airborne metagenome, as well as the application of targeted detection within this background, could provide a valuable molecular biosurveillance tool for monitoring potential threats to public health.

Acknowledgments This work was funded by the Department of Homeland Security and performed under the auspices of the U.S. Department of Energy by Lawrence Livermore National Laboratory under Contract DE-AC52-07NA27344. Maria Shin from Eureka Genomics is acknowledged for preparing sequencing libraries and generating the sequence data. Marilyn Ramsey, Anne Marie Erler, Gloria Murphy, Michael Dillon, Staci Kane, and Thomas Bunt from the LLNL environmental monitoring laboratory are acknowledged for their contribution toward collection and preparation of samples for this study.

Disclaimer This document was prepared as an account of work sponsored by an agency of the US government. Neither the US government nor Lawrence Livermore National Security, LLC, nor any of their employees make any warranty, expressed or implied, or assume any legal liability or responsibility for the accuracy, completeness, or usefulness of any information, apparatus, product, or process disclosed, or represent that its use would not infringe privately owned rights. Reference herein to any specific commercial product, process, or service by trade name, trademark, manufacturer, or otherwise does not necessarily constitute or imply its endorsement, recommendation, or favoring by the US government or Lawrence Livermore National Security, LLC. The views and opinions of authors expressed herein do not necessarily state or reflect those of the US government or Lawrence Livermore National Security, LLC, and shall not be used for advertising or product endorsement purposes.

Open Access This article is distributed under the terms of the Creative Commons Attribution License which permits any use, distribution, and reproduction in any medium, provided the original author(s) and the source are credited.

\section{References}

1. Monier JM, Demaneche S, Delmont TO, Mathieu A, Vogel TM, Simonet P (2011) Metagenomic exploration of antibiotic resistance in soil. Curr Opin Microbiol 14(3):229-235

2. Simon C, Daniel R (2011) Metagenomic analyses: past and future trends. Appl Environ Microbiol 77(4):1153-1161

3. Yooseph S, Sutton G, Rusch DB, Halpern AL, Williamson SJ, Remington K, Eisen JA, Heidelberg KB, Manning G, Li W, Jaroszewski L, Cieplak P, Miller CS, Li H, Mashiyama ST, Joachimiak MP, van Belle C, Chandonia JM, Soergel DA, Zhai Y, Natarajan K, Lee S, Raphael BJ, Bafna V, Friedman R, Brenner SE, 
Godzik A, Eisenberg D, Dixon JE, Taylor SS, Strausberg RL, Frazier M, Venter JC (2007) The Sorcerer II Global Ocean Sampling expedition: expanding the universe of protein families. PLoS Biol 5(3): e16

4. Philippot L, Raaijmakers JM, Lemanceau P, van der Putten WH (2013) Going back to the roots: the microbial ecology of the rhizosphere. Nat Rev Microbiol 11(11):789-799

5. Lighthart B, Shaffer BT (1995) Airborne bacteria in the atmospheric surface layer: temporal distribution above a grass seed field. Appl Environ Microbiol 61(4):1492-1496

6. Wilson SC, Morrow-Tesch J, Straus DC, Cooley JD, Wong WC, Mitlohner FM, McGlone JJ (2002) Airborne microbial flora in a cattle feedlot. Appl Environ Microbiol 68(7):3238-3242

7. Cronholm LS (1980) Potential health hazards from microbial aerosols in densely populated urban regions. Appl Environ Microbiol 39(1):6-12

8. Fang Z, Ouyang Z, Hu L, Wang X, Zheng H, Lin X (2005) Culturable airborne fungi in outdoor environments in Beijing, China. Sci Total Environ 350(1-3):47-58

9. Harrison RM, Jones AM, Biggins PD, Pomeroy N, Cox CS, Kidd SP, Hobman JL, Brown NL, Beswick A (2005) Climate factors influencing bacterial count in background air samples. Int $\mathrm{J}$ Biometeorol 49(3):167-178

10. Jones AM, Harrison RM (2004) The effects of meteorological factors on atmospheric bioaerosol concentrations - a review. Sci Total Environ 326(1-3):151-180

11. Qian J, Hospodsky D, Yamamoto N, Nazaroff WW, Peccia J (2012) Size-resolved emission rates of airborne bacteria and fungi in an occupied classroom. Indoor Air 22(4):339-351

12. Kembel SW, Jones E, Kline J, Northcutt D, Stenson J, Womack AM, Bohannan BJ, Brown GZ, Green JL (2012) Architectural design influences the diversity and structure of the built environment microbiome. ISME J 6(8):1469-1479

13. Adams RI, Miletto M, Taylor JW, Bruns TD (2013) Dispersal in microbes: fungi in indoor air are dominated by outdoor air and show dispersal limitation at short distances. ISME J 7(7):1262-1273

14. Bowers RM, McLetchie S, Knight R, Fierer N (2011) Spatial variability in airborne bacterial communities across land-use types and their relationship to the bacterial communities of potential source environments. ISME J 5(4):601-612

15. Bowers RM, Sullivan AP, Costello EK, Collett JL Jr, Knight R, Fierer N (2011) Sources of bacteria in outdoor air across cities in the midwestern United States. Appl Environ Microbiol 77(18):63506356

16. Wery N (2014) Bioaerosols from composting facilities - a review. Front Cell Infect Microbiol 4:42

17. Brandl H, Fricker-Feer C, Ziegler D, Mandal J, Stephan R, Lehner A (2014) Distribution and identification of culturable airborne microorganisms in a Swiss milk processing facility. J Dairy Sci 97(1):240 246

18. Martin E, Dziurowitz N, Jackel U, Schafer J (2014) Detection of airborne bacteria in a duck production facility with two different personal air sampling devices for an exposure assessment. J Occup Environ Hyg.

19. Smith DJ, Jaffe DA, Birmele MN, Griffin DW, Schuerger AC, Hee J, Roberts MS (2012) Free tropospheric transport of microorganisms from Asia to North America. Microb Ecol 64(4):973-985

20. Smith DJ, Timonen HJ, Jaffe DA, Griffin DW, Birmele MN, Perry KD, Ward PD, Roberts MS (2013) Intercontinental dispersal of bacteria and archaea by transpacific winds. Appl Environ Microbiol 79(4):1134-1139

21. Rodo X, Curcoll R, Robinson M, Ballester J, Burns JC, Cayan DR, Lipkin WI, Williams BL, Couto-Rodriguez M, Nakamura Y, Uehara R, Tanimoto H, Morgui JA (2014) Tropospheric winds from northeastern China carry the etiologic agent of Kawasaki disease from its source to Japan. Proc Natl Acad Sci U S A 111(22):7952-7957
22. Regan JF, Makarewicz AJ, Hindson BJ, Metz TR, Gutierrez DM, Corzett TH, Hadley DR, Mahnke RC, Henderer BD, Breneman JW, Weisgraber TH, Dzenitis JM (2008) Environmental monitoring for biological threat agents using the autonomous pathogen detection system with multiplexed polymerase chain reaction. Anal Chem 80(19):7422-7429

23. Broderick NA, Raffa KF, Handelsman J (2010) Chemical modulators of the innate immune response alter gypsy moth larval susceptibility to Bacillus thuringiensis. BMC Microbiol 10:129

24. Wang J, Van Nostrand JD, Wu L, He Z, Li G, Zhou J (2011) Microarray-based evaluation of whole-community genome DNA amplification methods. Appl Environ Microbiol 77(12):4241-4245

25. Han T, Chang CW, Kwekel JC, Chen Y, Ge Y, Martinez-Murillo F, Roscoe D, Tezak Z, Philip R, Bijwaard K, Fuscoe JC (2012) Characterization of whole genome amplified (WGA) DNA for use in genotyping assay development. BMC Genomics 13:217

26. McMurdie PJ, Holmes S (2013) Phyloseq: an R package for reproducible interactive analysis and graphics of microbiome census data. PLoS One 8(4):e61217

27. Rajaram S, Oono Y (2010) NeatMap - non-clustering heat map alternatives in R. BMC Bioinforma 11:45

28. Pinard R, de Winter A, Sarkis GJ, Gerstein MB, Tartaro KR, Plant RN, Egholm M, Rothberg JM, Leamon JH (2006) Assessment of whole genome amplification-induced bias through high-throughput, massively parallel whole genome sequencing. BMC Genomics 7:216

29. Direito SO, Zaura E, Little M, Ehrenfreund P, Roling WF (2014) Systematic evaluation of bias in microbial community profiles induced by whole genome amplification. Environ Microbiol 16(3):643-657

30. Abbai NS, Govender A, Shaik R, Pillay B (2011) Pyrosequence analysis of unamplified and whole genome amplified DNA from hydrocarbon-contaminated groundwater. Mol Biotechnol 50(1):3948

31. Ryan MP, Pembroke JT, Adley CC (2006) Ralstonia pickettii: a persistent gram-negative nosocomial infectious organism. J Hosp Infect 62(3):278-284

32. Han CS, Xie G, Challacombe JF, Altherr MR, Bhotika SS, Brown N, Bruce D, Campbell CS, Campbell ML, Chen J, Chertkov O, Cleland C, Dimitrijevic M, Doggett NA, Fawcett JJ, Glavina T, Goodwin LA, Green LD, Hill KK, Hitchcock P, Jackson PJ, Keim P, Kewalramani AR, Longmire J, Lucas S, Malfatti S, McMurry K, Meincke LJ, Misra M, Moseman BL, Mundt M, Munk AC, Okinaka RT, Parson-Quintana B, Reilly LP, Richardson P, Robinson DL, Rubin E, Saunders E, Tapia R, Tesmer JG, Thayer N, Thompson LS, Tice H, Ticknor LO, Wills PL, Brettin TS, Gilna P (2006) Pathogenomic sequence analysis of Bacillus cereus and Bacillus thuringiensis isolates closely related to Bacillus anthracis. J Bacteriol 188(9):3382-3390

33. Be NA, Thissen JB, Gardner SN, McLoughlin KS, Fofanov VY, Koshinsky H, Ellingson SR, Brettin TS, Jackson PJ, Jaing CJ (2013) Detection of Bacillus anthracis DNA in complex soil and air samples using next-generation sequencing. PLoS One 8(9):e73455

34. Leekha S, Diekema DJ, Perencevich EN (2012) Seasonality of staphylococcal infections. Clin Microbiol Infect 18(10):927-933

35. Whon TW, Kim MS, Roh SW, Shin NR, Lee HW, Bae JW (2012) Metagenomic characterization of airborne viral DNA diversity in the near-surface atmosphere. J Virol 86(15):8221-8231

36. Shade A, Jones SE, Caporaso JG, Handelsman J, Knight R, Fierer N, Gilbert JA (2014) Conditionally rare taxa disproportionately contribute to temporal changes in microbial diversity. MBio 5(4):e01371-14

37. Barberan A, Henley J, Fierer N, Casamayor EO (2014) Structure, inter-annual recurrence, and global-scale connectivity of airborne microbial communities. Sci Total Environ 487:187-195

38. Pankhurst LJ, Whitby C, Pawlett M, Larcombe LD, McKew B, Deacon LJ, Morgan SL, Villa R, Drew GH, Tyrrel S, Pollard SJ, Coulon F (2012) Temporal and spatial changes in the microbial bioaerosol communities in green-waste composting. FEMS Microbiol Ecol 79(1):229-239 